\title{
Treatment of the early stages of bipolar disorder
}

\author{
Thomas Elias Elanjithara, Sophia Frangou \& Philip McGuire
}

\begin{abstract}
SUMMARY
For many patients with bipolar disorder there is a long delay between the onset of illness and receiving a diagnosis and the initiation of treatment. This may have an adverse effect on the clinical outcome. Early intervention in bipolar disorder has received less attention than in schizophrenia, and there are relatively few specialist services in this area. This article reviews the literature on the early detection of bipolar disorder and on the effectiveness of pharmacological, psychological and psychosocial interventions in the early phase of the disorder.
\end{abstract}

\section{DECLARATION OF INTEREST}

None.

Current management strategies for bipolar disorder focus on the treatment of acute episodes, relapse prevention and maintenance therapies (National Institute for Health and Clinical Excellence 2006). To date, early intervention in bipolar disorder has received relatively little attention, in contrast to the increasing numbers of articles published in recent years on early intervention in schizophrenia.

Bipolar disorder affects about $1 \%$ of the population worldwide. A recent international study reported that the median age at onset is 24 years for men and 27 years for women, with a range of 10 to 42 years (Baldessarini 2009). In the majority of patients, bipolar disorder runs a chronic course, associated with significant morbidity in psychological, social and physical health (Kupfer 2005). The estimated cost of bipolar disorder in the UK is $£ 4.59$ billion (estimated 2007 value; Fajutrao 2009), including both direct costs such as those of hospitalisation for acute episodes, and indirect costs such as those of unemployment and suicide.

\section{The need for intervention in the early stages of bipolar disorder}

Arguably the most significant challenge is the timely recognition and treatment of the disorder. A large body of evidence across many different countries and service delivery systems has consistently reported delays of several years in making a diagnosis. In a study investigating history of illness prior to diagnosis in 240 patients with bipolar disorder, Berk et al (2007) found that participants first developed manic symptoms at a median age of 21 , and had their first full episode of mania at 24 . However, they did not receive a diagnosis of bipolar disorder until a median age of 30. Hirschfeld and colleagues (2003) had previously reported a delay of about 10 years from the first episode of illness to the first diagnosis.

An important difficulty in making an initial diagnosis is in differentiating bipolar disorder from a depressive disorder. Up to 89\% of the first episodes of illness in bipolar disorder are depressive (Duffy 2009a), and during the disorder's course (bipolar I and II: Box 1), depressive symptoms dominate the clinical picture (Benazzi 2006), so that milder manic or hypomanic episodes may be missed.

Bipolar-spectrum disorders, which include subthreshold mood states and milder manic presentations (Angst 2007), pose another challenge to early identification, as most patients do not seek help at that stage. Yet they represent a group that is likely to suffer functional impairment to varying degrees over time (Angst 2007). In addition, the current classification system may not be helpful in the identification of bipolar-spectrum disorders, as the majority of these presentations are classified under major depressive disorder (Angst 2007).

Mania is the diagnostic hallmark of bipolar disorder, but the presence of psychotic symptoms, especially in patients presenting with a manic onset, poses a diagnostic challenge. Psychotic symptoms may be present in $70-88 \%$ of patients

\section{BOX 1 Bipolar I and bipolar II disorder}

\section{Bipolar I disorder}

Characterised by at least one manic episode or mixed episode (clear manic and depressive features present in the same episode). There may also be a history of depressive episodes, although these are not necessary for establishing a diagnosis.

\section{Bipolar II disorder}

Characterised by one or more major depressive episodes, together with at least one hypomanic episode in the clinical course.

(American Psychiatric Association 1994)

\section{ARTICLE}

Thomas Elias Elanjithara is a higher specialty trainee in adult psychiatry at Barts and the London training scheme and an honorary research associate at the Institute of Psychiatry. His research interests include early intervention in mental health and psychosomatic disorders. Sophia Frangou is a reader in psychiatry at the Department of Psychosis Studies at the Institute of Psychiatry, King's College London (part of King's Health Partners Academic Health Sciences Centre) Her research focuses on the mechanisms of genetic vulnerability and disease expression in bipolar disorder. Philip McGuire is Head of the Department of Psychosis Studies at the Institute of Psychiatry, King's Health Partners Academic Health Sciences Centre. His research and clinical interests are in the mechanisms underlying the development of psychiatric disorders, and in early intervention. Correspondence Professor Philip McGuire, Institute of Psychiatry, Box P067, De Crespigny Park, London SE5 8AF, UK. Email: Philip. McGuire@kcl.ac.uk 
presenting with a manic onset (Tohen 2003; Yatham 2009). Additionally, in about $40 \%$ of such cases, the psychotic features are not mood congruent (Tohen 2003). Up to $20 \%$ of patients with psychotic features at the first presentation of mania, especially those with auditory hallucinations or first-rank symptoms, are misdiagnosed as having a non-affective psychotic disorder such as brief psychotic disorder, psychotic disorder not otherwise specified or schizophreniform disorder (Salvatore 2009). Particularly in younger patients, there is also the possibility of diagnostic confusion with attention-deficit hyperactivity disorder, personality disorders and substance use-related disorders (Berk 2009).

A delay in making a diagnosis of bipolar disorder has potential consequences for clinical outcome. Gazalle et al (2005) found that the number of undiagnosed years was directly associated with the level of subsequent psychopathology, particularly depression, and a poorer quality of life. Furthermore, the delay in initiation of mood stabilisers may be associated with subsequent poorer social functioning and increased risk of lifetime suicide attempts (Goldberg 2002). The present review examines the case for the development of disorder-specific pathways for early detection, and considers the priorities for the optimal treatment of the early stages of bipolar disorder.

\section{High-risk status and the prodromal stage of bipolar disorder}

\section{Genetic risk}

There have been attempts to identify groups that have a high risk of developing bipolar disorder, with

BOX 2 Features currently studied for endophenotype status in bipolar disorder

\begin{tabular}{ll}
\hline Neuroanatomical abnormalities & Neurophysiological abnormalities \\
- Amygdala & - Prepulse inhibition \\
- Ventral prefrontal cortex & - Auditory evoked P300 \\
- Cingulate & - Circadian rhythm \\
- Basal ganglia & Temperament and personality traits \\
- Hippocampus & - Cyclothymia \\
- White matter & - Hyperthymia \\
Neurocognitive abnormalities & - Behavioural disinhibition \\
- Verbal memory & \\
- Executive deficits & \\
- Response inhibition deficits & \\
- Visual spatial deficits & \\
- Facial emotion labelling deficits
\end{tabular}

a particular focus on individuals at genetic risk. In a large population-based study, Lichtenstein et al (2009) reported the relative risk of bipolar disorder in the offspring $(\mathrm{RR}=6.4)$ and siblings $(\mathrm{RR}=7.9)$ of probands with bipolar disorder. They also reported a heritability of $59 \%$ for bipolar disorder. Additionally, the offspring of probands with schizophrenia were found to have a relative risk of 5.2 for bipolar disorder. However, the majority of patients with bipolar disorder do not have a firstdegree family history (Correll 2007a).

\section{Biological markers of high-risk traits and transition to syndromal states}

Another strategy to identify high-risk status of developing bipolar disorder is detection of endophenotypes (Correll 2007a). Although they do not represent a prodromal state, endophenotypes are biological traits or markers indicating high risk for a specific disorder (Luby 2010). These traits are heritable, state-independent, cosegregate with illness within families and present in unaffected relatives at a higher rate than in the general population (Hasler 2006). Currently studied candidates for endophenotype status in bipolar disorder are shown in Box 2 (Correll 2007a; Luby 2010). Also being investigated are brain structural changes that may help with early identification of high-risk individuals who are more likely to undergo transition to syndromal states. A preliminary study comparing patients with bipolar I disorder and their unaffected relatives with major depressive disorder showed association of increased left insular volume with genetic predisposition, increased left substantia nigra volume with manic expression and increased left vermal volume with resilience to bipolar disorder (Kempton 2009).

\section{Clinical characteristics of the prodromal stage}

Recent studies have sought to define the prodromal stage of bipolar disorder and have highlighted the associated challenges in its recognition (Howes 2010). This stage can span several years, with subthreshold symptoms beginning in childhood and adolescence ('distal' or 'early' prodrome), which increase in number, frequency and severity ('proximal' or 'late' prodrome) immediately preceding the first episode of mania (Skjelstad 2009; Howes 2010). The mean duration of the proximal prodrome has been reported as 1.8-2.3 years (Correll 2007b).

Most of the published studies reporting on the prodromal phase have used retrospective design and lacked a control group. This limits sensitivity and specificity analysis, and an effect of recall bias cannot be excluded (Howes 2010). Correll et al 
(2007b), in a retrospective study on patients with bipolar I disorder using a structured assessment tool, reported a number of subthreshold manic and depressive symptoms and other symptoms in the prodromal phase. In a prospective study of a large community cohort, Tijssen and colleagues (2010) reported a dose-dependent relationship of persistent subthreshold hypomanic and depressive symptoms with 10-year clinical outcome of bipolar disorder. Although some of the prodromal symptoms (Box 3) have high specificity, all have relatively low sensitivity (Howes 2010). With a high specificity, they yield fewer false positives and can rule out cases as not bipolar disorder, thereby helping in considering the use of interventions that may have adverse effects, such as medications. However, a low sensitivity means that they capture fewer true positives and do not effectively rule in cases, which can be disadvantageous where psychosocial interventions (with less risk of adverse effects) may have a role. In some patients, the prodromal stage is associated with a significant functional decline (Skjelstad 2009). In the proximal prodromal phase there can be attenuated psychotic symptoms, particularly in those who subsequently develop mania with psychosis (Correll 2007b). The duration of these symptoms is much shorter than in the schizophrenia prodrome, occurring closer to the time of transition to full-blown bipolar disorder (Correll 2007b).

Duffy et al (2010), in a prospective study of highrisk offspring of patients with bipolar disorder, reported that there is a chronological sequence of symptoms, starting with anxiety and sleep disorders, followed by mood disturbances, which in association with comorbid substance misuse progress to full-blown mood episodes. It is not yet clear whether this also applies to patients with bipolar disorder without a family history of mood disorders. Cyclothymic features such as mood swings/lability are precursors of bipolar disorder (Angst 2003; Kochman 2005). However, it is not yet known whether these features constitute a personality disorder/trait or a prodromal state (Howes 2010). Thus, combining the findings of certain clinical features with the presence of other high-risk factors could help clinicians to better identify the prodromal state of bipolar disorder.

\section{Treatment challenges in the prodromal stage}

The potential role of clinical intervention in the prodromal stage of bipolar disorder is even less clear. There have been studies showing an improvement in mood symptoms with quetiapine (DelBello 2007) and in aggression with valproate (Saxena 2006) in individuals at high risk for the disorder, but there have also been trials with
BOX 3 List of frequently reported prodromal symptoms

\begin{tabular}{|c|c|}
\hline Subthreshold mania symptoms & Others \\
\hline $\begin{array}{l}\text { - Elevated/irritable mood lasting more than } \\
6 \mathrm{~h}_{\text {dday }}{ }^{\mathrm{a}}\end{array}$ & $\begin{array}{l}\text { - Mood swings (fluctuation between } \\
\text { elevated/irritable and depressive states) }\end{array}$ \\
\hline - Racing thoughts & - Sleep disturbances \\
\hline - Very rapid speech & - Anxiety symptoms ${ }^{a}$ \\
\hline - Increased energy & - Drop in functioning \\
\hline - Reckless/dangerous behaviour & - Decreased concentration \\
\hline Subthreshold depressive symptoms & - Social isolation \\
\hline - Depressed mood lasting more than & - Marked change in appetite \\
\hline $6 \mathrm{~h} /$ day $^{\mathrm{a}}$ & - Hearing voices ${ }^{\mathrm{a}}$ \\
\hline - Anhedonia & a. Features with a specificity $>80 \%$ and low \\
\hline - Self-harming ${ }^{a}$ & sensitivity \\
\hline - Suicidal thoughts ${ }^{\mathrm{a}}$ & (Howes 2010) \\
\hline
\end{tabular}

negative results (Salvadore 2008). Psychological treatments have an established role in reducing relapse in established bipolar disorder when used adjunctively (Scott 2007), and might also be useful in reducing the risk of illness in those at high risk of bipolar disorder, as has been reported in individuals at high risk of psychosis (Morrison 2004). An ongoing study in the high-risk population is comparing interpersonal psychotherapy with educational clinical monitoring (http:// clinicaltrials.gov/ct2/show/NCT00338806).

\section{Neuroprotection in the early stages of bipolar disorder}

Low doses of lithium have been reported to counteract hippocampal microstructural and metabolic changes, which correlated with the improvement of symptoms in individuals at ultrahigh risk for psychosis (Berger 2008; McGorry 2009a). This would be consistent with its effects on pathways involved in neuronal plasticity and integrity, particularly through upregulation of the neuroprotective protein Bcl-2 and BDNF (brainderived neurotrophic factor) genes and inhibition of pro-apoptosis glucose synthase kinase-3 signalling pathways (Zarate 2006; Schloesser 2008). In preclinical studies, lithium promotes neurogensis, consistent with evidence from clinical studies that it may be associated with the preservation of brain volume in key limbic and paralimbic regions implicated in bipolar disorder (Sassi 2004; Yucel 2008). Moore and colleagues (2009) reported that in patients with multi-episode bipolar disorder the neurotrophic changes induced by lithium were associated with clinical response. Quetiapine (Atmaca 2007) and valproate (Schloesser 2008) may also have neuroprotective potential, although the evidence for this is relatively limited. 


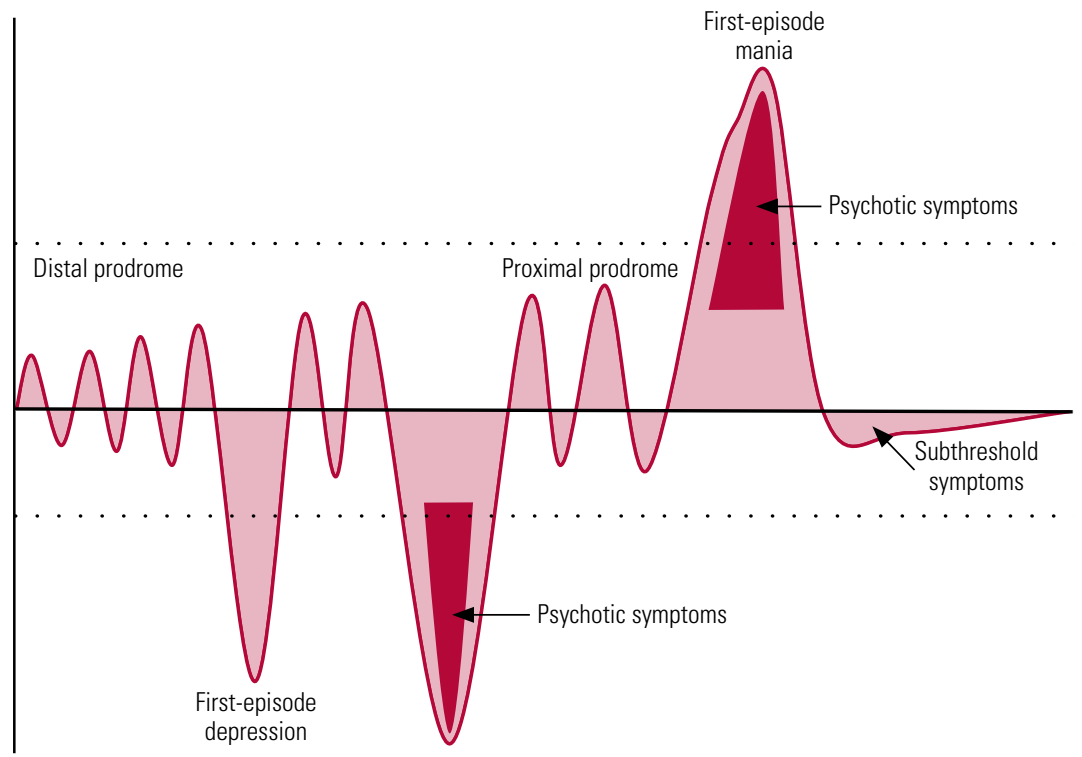

FIG 1

Simplified representation of the early stages of bipolar disorder. This figure does not include all clinical subgroups and presentations of the disorder. The shaded areas represent mood changes in relation to the euthymic state (black horizontal line) over a period of years spanning from teenage to early adulthood. The dotted lines indicate the current thresholds for diagnosis of manic and depressive episodes.

\section{The challenge of recognising a depressive onset in bipolar disorder}

The majority of patients with bipolar disorder initially present with a depressive episode (Fig. 1). Differentiating an episode of unipolar disorder from an episode of bipolar disorder (when there is no history of mania or hypomania) remains a challenge, despite substantial research efforts (Forty 2008). Although not pathognomonic, a number of features are more commonly seen in bipolar depression than in unipolar depression (Box 4) (Forty 2008; Mitchell 2008). They assist the clinician in a probabilistic approach to

BOX 4 Features indicative of bipolarity in depressive episodes

\begin{tabular}{ll}
\hline - Family history of bipolar disorder & melancholia \\
- Nature of the onset: & pathological guilt \\
$\begin{array}{l}\text { abrupt onset and offset of episode } \\
\text { antidepressant-induced mania, }\end{array}$ & lability of mood \\
hypomania or mixed states & irritability \\
younger age at onset of major depression & mixed depression (manic features during \\
postnatal onset & depressive episode) \\
- Symptom features: & Other features: \\
psychomotor retardation/agitation & comorbid substance misuse \\
atypical features such as & seasonality \\
hypersomnolence, hyperphagia, leaden & brief episodes of depression (<3 months) \\
paralysis & antidepressant wear-off (rapid \\
psychotic features & emergence of depressive symptoms after \\
& remission while on antidepressants)
\end{tabular}

considering bipolarity rather than helping to form a definite diagnostic tool.

There have been recent attempts to differentiate bipolar disorder depression from major depressive disorder by neuroimaging and neurocognitive studies. In a review of 140 imaging studies, Konarski et al (2008) reported enlarged striatal and amygdala volumes in multi-episode bipolar disorder compared with major depressive disorder. In addition, in a preliminary functional magnetic resonance imaging study, Almeida and colleagues (2010) reported elevated amygdala activation to sad facial expressions in patients with multiepisode bipolar disorder depression compared with patients with major depressive disorder. In a study comparing unmedicated patients with bipolar disorder (depressed state) with healthy controls (Roiser 2009), patients showed deficits mostly in emotion-dependent or 'hot' cognitive processing, rather than in emotion-independent or 'cold' cognitive processing. The deficits in the patients were apparent on tests involving reward processing, short-term memory storage and sensitivity to negative feedback. Taylor Tavares et al (2007) reported a broader range of executive impairments in patients with major depressive disorder than in patients with bipolar II depression. Analogous studies in the early stages of bipolar disorder have yet to be conducted.

A number of screening tools are available that may aid clinicians when faced with making a diagnosis in a young patient presenting with depression. These include self-report tools such as the Mood Disorder Questionnaire (Hirschfeld 2000), the Bipolar Spectrum Diagnostic Scale (Ghaemi 2005) and the 32-item Hypomania Checklist (HCL-32; Angst 2005). In multi-episode bipolar disorder, a score of 20 or more on the HCL32 yielded a sensitivity of $68 \%$ and specificity of $83 \%$ in distinguishing between bipolar disorder depression and major depressive disorder (Forty 2009). These instruments represent a potentially useful adjunct to conventional clinical assessment. Clinical work in individuals at high risk of schizophrenia has been facilitated by the introduction of specialised instruments for the assessment of prodromal symptoms (McGorry 2009b).

\section{Treatment challenges in the early depressive episodes of bipolar disorder}

Although there is a need for more reliable measures to identify bipolarity in a first episode presenting with depression (with no history of mania/ hypomania), little is known about the best course of treatment in such situations. The effects of antidepressants, mood stabilisers or psychological 
therapies used alone or in combination on the short- and long-term outcome in patients with this presentation is unclear. In a small-sample randomised study comparing paroxetine with a combination of paroxetine plus divalproex sodium in youths with depression at high genetic risk of bipolar disorder, Findling et al (2008) reported neither to be particularly effective in the long-term management of depressive symptoms.

For depression in patients with an established diagnosis of bipolar disorder, the latest guidelines (Goodwin 2009) suggest the use of quetiapine for early treatment effect. As quetiapine has unique antipsychotic and antidepressant properties (Calabrese 2005), it is particularly useful for depressive episodes with psychosis. Lamotrigine is also recommended by the guidelines for initial treatment.

In patients with a history of mania, the guidelines (Goodwin 2009) warn against the use of antidepressant monotherapy and advise on combining antidepressants with antimanic agents, owing to increased risk of switching to mania. Tricyclic antidepressants and dual-acting antidepressants such as venlafaxine carry more risk of switching to mania than selective serotonin reuptake inhibitors. When antidepressants are used, it is recommended that they be tapered and discontinued as soon as remission is achieved (Goodwin 2009).

Use of psychological treatments such as interpersonal therapy, cognitive-behavioural therapy (CBT) or family-focused therapy can shorten acute depressive episodes (Goodwin 2009). Maintenance treatment in the early stages of bipolar disorder is discussed later in this article.

\section{Treatment challenges in patients with a manic onset}

\section{Prognosis in the early stages of bipolar disorder}

Accumulating evidence has challenged the notion that, compared with schizophrenia, bipolar disorder is a more benign illness with a relatively good prognosis. Following the first manic episode, of syndromal recovery is indeed high, with 84-90\% patients achieving remission in 6 months (Tohen 2000; Conus 2006). However, nearly $15 \%$ patients may cycle into depression without recovering from the initial manic episode (Zarate 2001), especially if they had a mixed affective picture at presentation. Even when syndromal remission is achieved, up to $40 \%$ of patients report depressive and anxiety symptoms at 6 months after remission (Conus 2006). Furthermore, $40-50 \%$ of patients experience a recurrence within a year of disease onset, with time to a mood episode ranging from
6 to 8 months (Tohen 2003; Yatham 2009). In general, following a first manic episode, depressive episodes dominate (Box 5). Inter-episode intervals are shorter for depressive episodes and their recurrence rate is higher than for manic episodes (Yatham 2009). Medication non-adherence during the treatment for first episode of mania ranged from 37 to $65 \%$, which is considerably higher than that in patients who have had multiple episodes (Craig 2004; Yatham 2009).

The concept of recovery in bipolar disorder goes beyond symptomatic remission, and includes patients' function in their personal and social roles. In bipolar disorder, functional recovery is often poor, even after the first episode, with only $35 \%$ of patients recovering functionally by 6 months (Conus 2006) and 43\% by 2 years (Tohen 2003). Factors associated with poor functional recovery include younger age, substance misuse and presence of depressive symptoms. As in the case of patients with multi-episode bipolar disorder, even subsyndromal depressive symptoms contribute to functional impairment (Kauer-Sant'Anna 2009) following the first episode of mania.

The risk of attempted and completed suicide is of particular concern in the early stages of bipolar disorder. For example, in a prospective study, Khalsa (2008) reported 39 suicidal acts in 4 years in a cohort of 216 individuals. Of these, $87 \%$ occurred in the first year following disorder onset. An additional consideration is the high prevalence of comorbid disorders. Prevalence rates of substance misuse in this group range from 30 to $60 \%$. In a preliminary report on 4 -year follow-up of patients with first-episode bipolar I disorder, Baethge and colleagues (2008) reported that cannabis use selectively and strongly preceded and coincided with mania/hypomania, while a similar association was found between alcohol and
BOX 5 Factors that may be associated with increased risk of relapse following first-episode mania
Manic relapses
- Substance misuse
- Initial manic presentation, as opposed to mixed state
- Early age at onset
Depressive relapses
- Initial mixed $v$. manic presentation
- Substance misuse
- Higher occupational status
- Higher initial depression scores
- Early age at onset 
depressive recurrences. Additionally, substance misuse is a key predictor of non-adherence to treatment (Yatham 2009).

\section{Treatment of the first manic episode and subsequently}

\section{Treatment of first-episode mania}

At present it is not clear whether special provisions or considerations should apply for the treatment of the early stages of bipolar disorder that are distinct from those for multi-episode bipolar disorder. Current guidelines have no specific recommendations (American Psychiatric Association 2002; Hirschfeld 2005; Scottish Intercollegiate Guidelines Network 2005; National Institute for Health and Clinical Excellence 2006; Goodwin 2009). Empirical evidence suggests that currently available antipsychotics have antimanic properties (Perlis 2006). As in schizophrenia (Gafoor 2010), it is unclear whether some antipsychotics are more efficacious or better tolerated in the early stages of the illness than others. In a small retrospective medical record study of first-episode acute mania with psychosis, olanzapine was associated with better symptom remission and shorter time to remission than risperidone (Lambert 2005). Results are awaited from a recently concluded trial comparing a combination of lithium plus chlorpromazine with lithium plus olanzapine in first-episode mania with psychotic features (http://clinicaltrials.gov/ ct2/show/NCT00202293). A recently initiated clinical trial comparing quetiapine and lithium is examining functional and neurochemical brain changes in first-episode bipolar mania (http:// clinicaltrials.gov/ct2/show/NCT00609193).

As patients with bipolar disorder may be more vulnerable than those with schizophrenia to developing antipsychotic-induced movement disorders (Gao 2008), careful medication selection and dosing are indicated in the early stages of illness. The effective dose of antipsychotics is significantly lower for first-episode schizophrenia than for later episodes of chronic schizophrenia, and even lower for prodromal symptoms (Gafoor 2010). It is not clear whether the same applies to antipsychotic use in the early phases of bipolar disorder.

In a naturalistic setting, patients with affective psychosis received lower doses of antipsychotics at discharge and at 6-month follow-up than those with non-affective psychosis, despite no differences in the peak treatment dose (Zarate 2000). In contrast to antipsychotics, the doses of mood stabilisers required in first-episode mania do not appear to be different from those needed in an established bipolar illness.

\section{Maintenance therapy}

As in multi-episode bipolar disorder, key issues in the management of first-episode bipolar mania concern the prevention of relapses and the reduction of residual symptoms. The NICE guidelines suggest that maintenance therapy in patients with bipolar I disorder should be considered after the second episode, or after a manic episode if it involved significant risk and adverse consequences (National Institute for Health and Clinical Excellence 2006). Therefore in the majority of patients, provisions for their long-term pharmacological treatment should be made at the early stages of the illness. The medications that are the focus of current studies on the treatment of this stage of bipolar disorder are lithium, quetiapine and valproate.

\section{Lithium}

A first-episode mania study in a naturalistic setting reported a trend towards earlier syndromal recovery and a reduced risk of relapse of either polarity in patients treated with lithium, compared with valproate and antipsychotics (Tohen 2003). Lithium may also reduce the risk of suicide, selfharm and death from all causes in patients with mood disorders (Cipriani 2005). Lithium treatment appears to have only few and minor negative effects on cognition, as reported in a meta-analysis (Wingo 2009). Safety and tolerability of long-term lithium administration is well established in young patients, although its use in young women of reproductive age requires additional monitoring, as described in current guidelines (Scottish Intercollegiate Guidelines Network 2005; National Institute for Health and Clinical Excellence 2006). The relatively high rate of medication nonadherence in the early stages of bipolar disorder may be particularly relevant for lithium, as sudden discontinuation could induce rebound (Maj 2003).

\section{Quetiapine}

In addition to its antimanic properties, quetiapine also has antidepressant properties (Calabrese 2005). Quetiapine treatment is shown to be effective across a broad range of mood symptoms and the drug is well tolerated in multi-episode bipolar disorder (Vieta 2005). In a small openlabel study, Duffy et al (2009b) reported that quetiapine was also effective and well tolerated in a relatively young population with bipolar disorder with intact neurocognitive function. A recently initiated study is comparing quetiapine with lithium on neuroanatomical, neurochemical, neurocognitive and prophylactic effects after firstepisode mania (www.psychiatry.unimelb.edu.au/ centres-units/mnc/projects/mania.html). 
Valproate

Valproate maintenance has been associated with fewer depressive relapses in multi-episode bipolar disorder than lithium maintenance (Smith 2007). Although the overall effect of valproate as maintenance therapy in multi-episode bipolar disorder is less compelling (Vieta 2007), it is not clear what effect it has in the maintenance phase after the first episode. Also, valproate's teratogenic potential limits its use in female patients in the early stages of bipolar disorder.

\section{Psychoeducation and psychological interventions}

The high rate of medication non-adherence in the early stages of bipolar disorder emphasises the need to combine pharmacological treatment with psychoeducation. Psychoeducation is particularly effective in improving clinical outcomes by reducing non-adherence in patients with multiepisode bipolar disorder (Vieta 2004). Family psychoeducation can be more effective in the early stages of bipolar disorder than in the later stages, with improvement in time to recurrence (Reinares 2009). Comorbid substance misuse also requires management from the early stages (Baethge 2008). Cognitive-behavioural therapy may be helpful, with a focus on stress management and relapse prevention. Gleeson et al (2009) conducted a randomised controlled trial of multimodal individual and family CBT for relapse prevention in a first-episode psychosis group, which showed promising results in comparison with standard case management. Jones $\&$ Burrell-Hodgson (2008)reported the use of manualised CBT, with results showing improvement in circadian activity, prodromal coping skills and cognitive style in patients after their first episode of bipolar disorder.

\section{Directions for the future}

There is clearly a need for more research on the characteristics of the early stages of bipolar disorder and on the development of treatments that can be implemented in the early phases. There is also a need for the development of validated rating instruments for better identification of the prodromal phase, and studies to evaluate the utility of interventions aimed at preventing transition to a full-blown disorder from a highrisk state. Specialised service organisation and provisions may improve the early detection of bipolar disorder and facilitate research into the early intervention in bipolar disorder, as they have done for schizophrenia. Analogous services and interventions need to be evaluated for their impact on the outcome of bipolar disorder. Some
B0X 6 Priorities in the treatment of the early stages of bipolar disorder

- Enhancement of early detection and diagnosis

- Mood stabilisation (including treatment of subsyndromal mood symptoms)

- Neuroprotection

- Preservation of neurocognition

- Management of substance misuse and other comorbidities

- Improvement of medication adherence

- Management of risk

- Devising and administering appropriate pharmacological and psychological interventions

- Improvement in functional/educational/vocational outcomes

- Maintaining an optimistic treatment setting

key diagnostic and treatment priorities for early intervention services for bipolar disorder are suggested in Box 6.

\section{References}

Almeida JR, Versace A, Hassel S, et al (2010) Elevated amygdala activity to sad facial expressions: a state marker of bipolar but not unipolar depression. Biological Psychiatry 67: 414-21.

American Psychiatric Association (1994) Diagnostic and Statistical Manual of Mental Disorders (4th edn) (DSM-IV). APA.

American Psychiatric Association (2002) Practice Guideline for the Treatment of Patients with Bipolar Disorder, Second Edition. APA (http://www.psychiatryonline.com/pracGuide/PracticePDFs/Bipolar2e_ Inactivated_04-16-09.pdf).

Angst J, Gamma A, Endrass J (2003) Risk factors for the bipolar and depression spectra. Acta Psychiatrica Scandinavica 108 (suppl 418): $15-9$.

Angst J, Adolfsson R, Benazzi F, et al (2005) The HCL-32: towards a self-assessment tool for hypomanic symptoms in outpatients. Journal of Affective Disorders 88: 217-33.

Angst J (2007) The bipolar spectrum. British Journal of Psychiatry 190: 189-91.

Atmaca M, Ozdemir H, Cetinkaya S, et al (2007) Cingulate gyrus volumetry in drug free bipolar patients and patients treated with valproate or valproate and quetiapine. Journal of Psychiatric Research 41: 821-7.

Baethge C, Hennen J, Khalsa HM, et al (2008) Sequencing of substance use and affective morbidity in 166 first-episode bipolar I disorder patients. Bipolar Disorders 10: 738-41.

Baldessarini R, Bolzani L, Cruz N, et al (2009) Onset-age of bipolar disorders at six international sites. Journal of Affective Disorders 121: 143-6.

Benazzi $F$ (2006) Mood patterns and classification in bipolar disorder. Current Opinion in Psychiatry 19: 1-8.

Berger G, Wood S, Dell'olio M, et al (2008) Neuroprotective effects of low dose lithium in individuals at ultra-high risk for psychosis. Schizophrenia Research 102: 39-40.

Berk M, Dodd S, Callaly P, et al (2007) History of illness prior to a diagnosis of bipolar disorder or schizoaffective disorder. Journal of Affective Disorders 103: 181-6. 
Berk M, Malhi, Gin S, et al (2009) Early intervention in bipolar disorders: clinical, biochemical and neuroimaging imperatives. Journal of Affective Disorders 114: 1-3.

Calabrese JR, Keck Jr PE, Macfadden W, et al (2005) A randomized, double-blind, placebo-controlled trial of quetiapine in the treatment of bipolar I or II depression. American Journal of Psychiatry 162: 1351-60.

Cipriani A, Pretty $\mathrm{H}$, Hawton $\mathrm{K}$, et al (2005) Lithium in the prevention of suicidal behavior and all-cause mortality in patients with mood disorders: a systematic review of randomized trials. American Journal of Psychiatry 162: 1805-19.

Conus P, Cotton S, Abdel-Baki A, et al (2006) Symptomatic and functional outcome 12 months after a first episode of psychotic mania: barriers to recovery in a catchment area sample. Bipolar Disorders 8: 221-31.

Correll CU, Penzner JB, Lencz T, et al (2007a) Early identification and highrisk strategies for bipolar disorder. Bipolar Disorders 9: 324-38.

Correll CU, Penzner JB, Frederickson AM, et al (2007b) Differentiation in the preonset phases of schizophrenia and mood disorders: evidence in support of a bipolar mania prodrome. Schizophrenia Bulletin 33: 703-14.

Craig TJ, Grossman S, Mojtabai R, et al (2004) Medication use patterns and 2-year outcome in first-admission bipolar disorder with psychotic features. Bipolar Disorders 6: 406-15.

DelBello MP, Adler CM, Whitsel RM, et al (2007) A 12-week single-blind trial of quetiapine for the treatment of mood symptoms in adolescents at high risk for developing bipolar I disorder. Journal of Clinical Psychiatry 68: 789-95

Duffy A, Alda M, Hajek T, et al (2009a) Early course of bipolar disorder in high-risk offspring: prospective study. British Journal of Psychiatry 195: 457-8.

Duffy A, Milin R, Grof P (2009b) Maintenance treatment of adolescent bipolar disorder: open study of effectiveness and tolerability of quetiapine. BMC Psychiatry 9: 4.

Duffy A, Alda M, Hajek T, et al (2010) Early stages in the development of bipolar disorder. Journal of Affective Disorder 121: 127-35.

Fajutrao L, Locklear J, Priaulx J, et al (2009) A systematic review of the evidence of the burden of bipolar disorder in Europe. Clinical Practice and Epidemiology in Mental Health 5: 3.

Findling RL, Lingler J, Rowles BM, et al (2008) A pilot pharmacotherapy trial for depressed youths at high genetic risk for bipolarity. Journal of Child Adolescent Psychopharmacology 8: 615-21.

Forty L, Smith D, Jones L, et al (2008) Clinical differences between bipolar and unipolar depression. British Journal of Psychiatry 192: 388-9.

Forty L, Smith D, Jones L, et al (2009) Identifying hypomanic features in major depressive disorder using the Hypomania Checklist (HCL-32). Journal of Affective Disorders 114: 68-73.

Gafoor R, Landau S, Craig TK, et al (2010) Efficacy and adverse effects of quetiapine versus risperidone in first-episode schizophrenia. Journal of Clinical Psychopharmacology 30: 600-6.

Gao K, Kemp DE, Ganocy SJ, et al (2008) Antipsychotic-induced extrapyramidal side effects in bipolar disorder and schizophrenia: a systematic review. Journal of Clinical Psychopharmacology 28: 203-9.

Gazalle F, Andreazza A, Ceresér K, et al (2005) Clinical impact of late diagnose of bipolar disorder. Journal of Affective Disorders 86: 313-6.

Ghaemi SN, Miller C, Berv D, et al (2005) Sensitivity and specificity of a new bipolar spectrum diagnostic scale. Journal of Affective Disorders 84: $273-7$.

Gleeson JF, Cotton SM, Alvarez-Jimenez M, et al (2009) A randomized controlled trial of relapse prevention therapy for first-episode psychosis patients. Journal of Clinical Psychiatry 70: 477-86.

Goldberg JF, Ernst CL (2002) Features associated with the delayed initiation of mood stabilizers at illness onset in bipolar disorder. Journal of Clinical Psychiatry 63: 985-91.

Goodwin GM (2009) Evidence-based guidelines for treating bipolar disorder: revised second edition - recommendations from the British Association for Psychopharmacology. Journal of Psychopharmacology 23: $346-88$
Hasler G, Drevets WC, Gould TD, et al (2006) Toward constructing an endophenotype strategy for bipolar disorders. Biological Psychiatry 60: 93-105

Hirschfeld RM, Williams J, Spitzer RL, et al (2000) Development and validation of a screening instrument for bipolar spectrum disorder: the Mood Disorder Questionnaire. American Journal of Psychiatry 157: 1873-5.

Hirschfeld RM, Lewis L, Vornik LA (2003) Perceptions and impact of bipolar disorder: how far have we really come? Results of the National Depressive and Manic-Depressive Association 2000 survey of individuals with bipolar disorder. Journal of Clinical Psychiatry 64: 161-74.

Hirschfeld RMA (2005) Guideline Watch: Practice Guideline for the Treatment of Patients With Bipolar Disorder. American Psychiatric Association (http://www.psych.org/psych_pract/treatg/pg/prac_guide. cfm).

Howes OD, Lim S, Theologos G, et al (2010) A comprehensive review and model of putative prodromal features of bipolar affective disorder. Psychological Medicine Sep 14: 1-11. Epub ahead of print.

Jones SH, Burrell-Hodgson G (2008) Cognitive-behavioural treatment of first diagnosis bipolar disorder. Clinical Psychology and Psychotherapy 15: $367-77$

Kauer-Sant'Anna M, Bond DJ, Lam RW, et al (2009) Functional outcomes in first-episode patients with bipolar disorder: a prospective study from the Systematic Treatment Optimization Program for Early Mania project. Comprehensive Psychiatry 50: 1-8.

Kempton MJ, Haldane M, Frangou S, et al (2009) Dissociable brain structural changes associated with predisposition, resilience, and disease expression in bipolar disorder. Journal of Neuroscience 29: 10863-8.

Khalsa HMK, Salvatore P, Hennen J, et al (2008) Suicidal events and accidents in 216 first-episode bipolar I disorder patients: predictive factors. Journal of Affective Disorders 106: 179-84.

Kochman F, Hantouche E, Ferrari P, et al (2005) Cyclothymic temperament as a prospective predictor of bipolarity and suicidality in children and adolescents with major depressive disorder. Journal of Affective Disorders 85: 181-9.

Konarski JZ, Mclntyre RS, Kennedy SH, et al (2008) Volumetric neuroimaging investigations in mood disorders: bipolar disorder versus major depressive disorder. Bipolar Disorders 10: 1-37.

Kupfer DJ (2005) The increasing medical burden in bipolar disorder. JAMA 293: 2528-30.

Lambert M, Conus P, Schimmelmann BG, et al (2005) Comparison of olanzapine and risperidone in 367 first-episode patients with nonaffective or affective psychosis: results of an open retrospective medical record study. PharmacoPsychiatry 38: 206-13.

Lichtenstein P, Yip B , Björk C, et al (2009) Common genetic determinants of schizophrenia and bipolar disorder in Swedish families: a populationbased study. Lancet 373: 234-9.

Luby JL, Navsaria N (2010) Pediatric bipolar disorder: evidence for prodromal states and early markers. Journal of Child Psychology and Psychiatry 51: 459-71.

Maj M (2003) The effect of lithium in bipolar disorder: a review of the research evidence. Bipolar Disorders 5: 180-8.

McGorry PD, Yung AR, Pantelis C, et al (2009a) A clinical trials agenda for testing interventions in earlier stages of psychotic disorders. Medical Journal of Australia 190 (suppl 4): S33-6.

McGorry PD, Nelson B, Amminger GP, et al (2009b) Intervention in individuals at ultra high risk for psychosis: a review and future directions. Journal of Clinical Psychiatry 70: 1206-12.

Mitchell PB, Goodwin GM, Johnson GF, et al (2008) Diagnostic guidelines for bipolar depression: a probabilistic approach. Bipolar Disorders 10: $144-52$

Moore GJ, Cortese BM, Glitz DA, et al (2009) A longitudinal study of the effects of lithium treatment on prefrontal and subgenual prefrontal gray matter volume in treatment-responsive bipolar disorder patients. Journal of Clinical Psychiatry 70: 699-705 
Morrison AP, French P, Walford L, et al (2004) Cognitive therapy for the prevention of psychosis in people at ultra-high risk: randomised controlled trial. British Journal of Psychiatry 185: 291-7.

National Institute for Health and Clinical Excellence (2006) Bipolar Disorder: The Management of Bipolar Disorder in Adults, Children and Adolescents, in Primary and Secondary Care. NICE.

Perlis RH, Welge JA, Vornik LA, et al (2006) Atypical antipsychotics in the treatment of mania: a meta-analysis of randomized, placebo-controlled trials. Journal of Clinical Psychiatry 67: 509-16.

Reinares M, Colom F, Rosa A, et al (2009) The impact of staging bipolar disorder on treatment outcome of family psychoeducation. Journal of Affective Disorders 123: 81-6. Epub ahead of print.

Roiser JP, Cannon DM, Gandhi SK, et al (2009) Hot and cold cognition in unmedicated depressed subjects with bipolar disorder. Bipolar Disorders 11: 178-89.

Salvadore G, Drevets WC, Henter ID, et al (2008) Early intervention in bipolar disorder, part II: therapeutics. Early Intervention in Psychiatry 2: $136-46$.

Salvatore P, Baldessarini RJ, Tohen M, et al (2009) McLean-Harvard International First-Episode Project: two-year stability of DSM-IV diagnoses in 500 first-episode psychotic disorder patients. Journal of Clinical Psychiatry 70: 458-66.

Sassi RB, Brambilla P, Hatch JP, et al (2004) Reduced left anterior cingulate volumes in untreated bipolar patients. Biological Psychiatry 56: 467-75.

Saxena K, Howe M, Simeonova D, et al (2006) Divalproex sodium reduces overall aggression in youth at high risk for bipolar disorder. Journal of Child and Adolescent Psychopharmacology 16: 252-9.

Schloesser RJ, Huang J, Klein PS, et al (2008) Cellular plasticity cascades in the pathophysiology and treatment of bipolar disorder. Neuropsychopharmacology 33: 110-33.

Scott J, Colom F, Vieta E (2007) A meta-analysis of relapse rates with adjunctive psychological therapies compared to usual psychiatric treatment for bipolar disorders. International Journal of Neuropsychopharmacology 10: 123-9.

Scottish Intercollegiate Guidelines Network (2005) Bipolar Affective Disorder: A National Clinical Guideline (Guideline 82). SIGN (http://www. sign.ac.uk/guidelines/fulltext/82).

Skjelstad DV, Malt UF, Holte A (2009) Symptoms and signs of the initial prodrome of bipolar disorder: a systematic review. Journal of Affective Disorders 126: 1-13. Epub ahead of print.

Smith LA, Cornelius V, Warnock A, et al (2007) Effectiveness of mood stabilizers and antipsychotics in the maintenance phase of bipolar disorder: a systematic review of randomized controlled trials. Bipolar Disorders 9: 394-412.

Taylor Tavares JV, Clark L, Cannon DM, et al (2007) Distinct profiles of neurocognitive function in unmedicated unipolar depression and bipolar II depression. Biological Psychiatry 62: 917-24.

Tijssen MJA, van Os J, Wittchen HU, et al (2010) Prediction of transition from common adolescent bipolar experiences to bipolar disorder: 10-year study. British Journal of Psychiatry 196: 102-8.

Tohen M, Hennen J, Zarate Jr C, et al (2000) Two-year syndromal and functional recovery in 219 cases of first-episode major affective disorder with psychotic features. American Journal of Psychiatry 157: 220-8.

Tohen M, Zarate Jr CA, Hennen J, et al (2003) The McLean-Harvard First-Episode Mania Study: prediction of recovery and first recurrence. American Journal of Psychiatry 160: 2099-107.

Vieta E, Colom F (2004) Psychological interventions in bipolar disorder: from wishful thinking to an evidence-based approach. Acta Psychiatrica Scandinavica 110: 34-8.

Vieta E, Mullen J, Brecher M, et al (2005) Quetiapine monotherapy for mania associated with bipolar disorder: combined analysis of two international, double-blind, randomised, placebo-controlled studies. Current Medical Research and Opinion 21: 923-34.

Vieta E, Rosa AR (2007) Evolving trends in the long-term treatment of bipolar disorder. World Journal of Biological Psychiatry 8: 4-11.

Wingo AP, Wingo TS, Harvey PD, et al (2009) Effects of lithium on cognitive performance: a meta-analysis. Journal of Clinical Psychiatry 70: 1588-97.

Yatham LN, Kauer-Sant'Anna M, Bond DJ, et al (2009) Course and outcome after the first manic episode in patients with bipolar disorder: prospective 12-month data from the systematic treatment optimization program for early mania project. Canadian Journal of Psychiatry 54 : 105-12.

Yucel K, Taylor VH, McKinnon MC, et al (2008) Bilateral hippocampal volume increase in patients with bipolar disorder and short-term lithium treatment. Neuropsychopharmacology 33: 361-7.

Zarate Jr CA, Tohen M (2000) Antipsychotic drug treatment in firstepisode mania: a 6-month longitudinal study. Journal of Clinical Psychiatry 61: 33-8.

Zarate Jr CA, Tohen M, Fletcher $\mathrm{K}$ (2001) Cycling into depression from a first episode of mania: a case-comparison study. American Journal of Psychiatry 158: 1524-6.

Zarate Jr CA, Singha J, Manji HK (2006) Cellular plasticity cascades: targets for the development of novel therapeutics for bipolar disorder. Biological Psychiatry 59: 1006-20.

\section{MCOs}

Select the single best option for each question stem

1 In bipolar disorder:

a peak age at onset is after 40 years

b the illness runs a chronic course in the majority

c the illness is usually diagnosed early

$\mathrm{d}$ delay in diagnosis is not associated with worse outcome

e a manic picture dominates the overall clinical picture.

2 In the prodromal phase and high-risk groups:

a the majority of patients with bipolar disorder have an affected first-degree relative

b prodromal symptoms have high sensitivity

c lithium may have a protective role

d psychological therapies have been shown to be ineffective e substance misuse does not influence the course.

3 Regarding the first mood episode in bipolar disorder:

a the first episode is usually manic

b depressive first episodes are easy to identify as part of bipolar disorder

c certain clinical features may help to suspect bipolarity in patients with depression

$\mathrm{d}$ psychosis is rare in first-episode mania

e patients with first-episode mania always have manic relapses.

4 Prognosis following first-episode mania:

a the majority attain functional recovery quickly

b substance misuse is associated with poor medication adherence

\section{MCO answers}

$\begin{array}{lllll}1 \mathrm{~b} & 2 \mathrm{c} & 3 \mathrm{c} & 4 \mathrm{~b} & 5 \mathrm{a}\end{array}$ 